\title{
Exercise pre-conditioning alleviates brain damage via excitatory amino acid transporter 2 and extracellular signal-regulated kinase $1 / 2$ following ischemic stroke in rats
}

\author{
XIAO WANG ${ }^{1 *}$, MIN ZHANG $^{2 *}$, RUI FENG $^{1 *}$, WEN-BIN LI ${ }^{2}$, SHI-QING REN ${ }^{1}$ and FENG ZHANG ${ }^{3,4}$ \\ ${ }^{1}$ Department of Neurology, The Third Hospital of Hebei Medical University, Shijiazhuang, Hebei 050051; \\ ${ }^{2}$ Department of Pathophysiology, Hebei Medical University, Shijiazhuang, Hebei 050017; \\ ${ }^{3}$ Department of Rehabilitation Medicine; ${ }^{4}$ Hebei Provincial Orthopedic Biomechanics Key Laboratory, \\ The Third Hospital of Hebei Medical University, Shijiazhuang, Hebei 050051, P.R. China
}

Received February 1, 2014; Accepted September 29, 2014

DOI: $10.3892 / \mathrm{mmr} .2014 .2834$

\begin{abstract}
Previous studies have reported that physical exercise may exert a neuroprotective effect in humans as well as animals. However, the detailed mechanisms underlying the neuroprotective effect of exercise has remained to be elucidated. The aim of the present study was to explore the possible signaling pathways involved in the protective effect of pre-ischemic treadmill training for ischemic stroke in rats. A total of 36 male Sprague-Dawley rats were divided at random into three groups as follows ( $\mathrm{n}=12$ for each): Sham surgery group; middle cerebral artery occlusion (MCAO) group; and exercise with MCAO group. Following treadmill training for three weeks, the middle cerebral artery was occluded for $90 \mathrm{~min}$ in order to induce ischemic stroke, followed by reperfusion. Following $24 \mathrm{~h}$ post-reperfusion, six rats from each group were assessed for neurological deficits and then sacrificed to calculate the infarct volume. The remaining rats ( $\mathrm{n}=6$ for each group) were sacrificed and the expression levels of excitatory amino acid transporter 2 (EAAT-2) and extracellular signal-regulated kinase 1/2 (ERK1/2) were detected using western blot analysis. The results of the present study demonstrated that rats that underwent pre-ischemic exercise intervention had a significantly decreased brain infarct volume and neurological deficits; in addition, the pre-ischemic exercise group showed decreased overexpression of phosphorylated ERK1/2 and increased expression of EAAT-2 following ischemic stroke. In conclusion, treadmill training exercise prior to
\end{abstract}

Correspondence to: Dr Feng Zhang, Department of Rehabilitation Medicine, The Third Hospital of Hebei Medical University, 139 Ziqiang Road, Shijiazhuang, Hebei 050051, P.R. China

E-mail: zjk20019@126.com

*Contributed equally

Key words: middle cerebral artery occlusion, excitatory amino acid transporters, extracellular signal-regulated kinase $1 / 2$ ischemic stroke alleviated brain damage in rats via regulation of EAAT-2 and ERK1/2.

\section{Introduction}

Previous studies have demonstrated that physical exercise had neuroprotective effects in animals following ischemic stroke, including enhanced survival, decreased neurological deficits, reduced blood-brain barrier (BBB) dysfunction and increased neurovascular integrity (1-5). However, the mechanism underlying the neuroprotective effect of exercise have remained to be elucidated.

Excessive release of glutamate has been confirmed to have a key role in the process of brain damage following ischemic stroke and is involved in the majority of ischemic stroke-associated brain injuries (6). A previous study reported that three weeks of pre-ischemic treadmill training may ameliorate the increased release of glutamate (7) and decrease messenger (m)RNA levels of metabotropic glutamate receptor 5 and $N$-methyl-D-aspartate receptor subunit type $2 \mathrm{~B}(8)$, which resulted in alleviation of the toxic effects of excessive glutamate.

Glutamate transporters also have an important role in glutamate re-uptake, which contribute to the pathological mechanisms of glutamate-induced toxic injury. Five excitatory amino acid transporters (EAATs) have been identified in the mammalian CNS, of which EAAT-2 was an important transporter for the regulation of extracellular glutamate concentrations $(9,10)$. Therefore, the hypothesis of the present study was that pre-ischemic exercise intervention promotes the expression of EAAT-2 so as to reduce brain damage following ischemic stroke.

The extracellular signal-regulated kinase 1/2 (ERK1/2) pathway has been reported to have an important role in the neuroprotective effect of exercise preconditioning (11). ERK1/2 is a key subfamily of mitogen-activated protein kinases which regulate a broad range of cellular activities, including the protection against cerebral ischemia (12). Inhibition of the ERK1/2 pathway was previously reported to decrease brain damage and infarct volume in mice following 
ischemic stroke $(13,14)$; in addition, another study showed that following three weeks of pre-ischemic exercise the expression of phosphorylated (phospho)-ERK1/2 was downregulated $48 \mathrm{~h}$ post-reperfusion (11). However, the effect of pre-ischemic exercise on the expression of phospho-ERK1/2 at $24 \mathrm{~h}$ post-reperfusion has remained to be elucidated.

The aim of the present study was to investigate whether three weeks of exercise preconditioning reduced neurological deficits, infarct size as well as altered the expression of phospho-ERK1/2 and EAAT-2.

\section{Materials and methods}

Animals. A total of 36 male Sprague-Dawley rats (weight, 200-220g; age, 2 months) were provided by the Hebei Province Laboratory Animal Center (Hebei, China). Rats were housed under standard conditions in a 12-h light/dark cycle, with food and water available ad libitum. All procedures in the present study were approved by the Animal Care and Use Committee of Hebei Medical University (Hebei, China).

Treadmill training. Rats were divided at random into three groups as follows ( $\mathrm{n}=12$ for each): Sham surgery group; middle cerebral artery occlusion (MCAO) group; and exercise with MCAO group. Prior to formal training, rats in the exercise with MCAO group were subjected to running exercise for three days at a speed of 6-7 m/min for 20 min per day using a treadmill training machine (DSPT-202 Type 5-Lane Treadmill; Litai Biotechnology Co., Ltd, Shishi, China). Following adaptive exercise training, rats underwent formal exercise intervention at a speed of $20 \mathrm{~m} / \mathrm{min}$ for $30 \mathrm{~min}$ per day, six times per week. Rats in the sham surgery and MCAO groups were enabled to run freely in their cages in the corresponding period.

MCAO model. Following treadmill training, all rats underwent MCAO or sham surgery. The MCAO model rats were anesthetized (Sigma-Aldrich, Poole, UK) using an intraperitoneal injection of $4 \%$ chloral hydrate $(10 \mathrm{ml} / \mathrm{kg})$; further doses were required if rats did not remain anesthetized during surgery. A heating pad was used to maintain the rats' body temperature at $37^{\circ} \mathrm{C}$. Surgical procedures were performed as previously described by Longa et al (15).

Inbrief,the leftcommon carotid artery (CCA), externalcarotid artery (ECA) and internal carotid artery (ICA) were exposed. A suture (4-0 nylon) with a rounded poly-L-lysine-coated tip (Beijing Sunbio Biotech Co., Ltd, Beijing, China) was inserted into the small incision in the ECA. The suture was then passed through CCA and ICA to the origin section of the MCA in order to occlude this artery. Following 90 minutes of occlusion, the filament was removed to allow for reperfusion.

Rats in the sham group underwent the same procedures without occlusion of MCA. The physiological parameters of rats were determined using a Blood Gas and Electrolyte System (ABL505; Radiometer, Copenhagen, Denmark). Rats were evaluated $24 \mathrm{~h}$ following reperfusion in accordance with a widely used scale as follows: 0 , no neurological symptoms; 1 , unable to completely extend the front jaw on the contralateral side; 2 , rotating while crawling and falling to the contralateral side; 3 , unable to walk without assistance; and 4 , unconsciousness (15).
Determination of brain infarct volume. Following $24 \mathrm{~h}$ post-reperfusion, rats were anesthetized using chloral hydrate $(10 \%)$ and then sacrificed. Brain tissues were stored at $-20^{\circ} \mathrm{C}$ for 10 minutes, then the whole brain was divided evenly into six coronal sections $(2 \mathrm{~mm})$ from the anterior pole to the optic chiasm in the mid-way. Brain sections were immediately dipped into a 2\% 2,3,5-triphenyltetrazolium chloride solution (Sigma-Aldrich) at $37^{\circ} \mathrm{C}$ for $30 \mathrm{~min}$ and then fixed in $4 \%$ paraformaldehyde buffer (Sigma, Muenchen, Germany). Following 24 h, the infarct area was calculated according to the images captured using a digital camera (DC240; Kodak, Rochester, NY, USA) and imaging software (Adobe Photoshop 7.0; Adobe Systems, San Jose, CA, USA). The infarction volume of whole brain was equal to the sum of the infarct area in the six sections. In order to reduce error due to brain edema, the corrected formula to calculate the infarct volume was as follows (16): Infarct volume = contralateral hemisphere region - non-infarcted region in the ipsilateral hemisphere; infarct percentage $=($ Infarct volume/volume of the contralateral hemisphere)x $100 \%$.

Western blot analysis. Cortex brain tissue in proximity to the ischemic area was examined. The protein of the brain tissue was extracted using a protein extraction reagent (Pierce Biotechnology, Inc., Rockford, IL, USA). The extracted proteins were purified by affinity chromatography and the concentration of extracted protein was detected using the bicinchoninic acid assay (Beyotime Institute of Biotechnology, Haimen, China). Equal quantities $(40 \mu \mathrm{g})$ of protein extract and sample buffer were mixed completely and incubated in a $95^{\circ} \mathrm{C}$-water bath for 5 min prior to loading onto $10 \%$ polyacrylamide gels. The proteins were delivered onto a Hybond ${ }^{\mathrm{TM}}$ nylon membrane (GE Healthcare, Little Chalfont, UK) at $350 \mathrm{~V}$ for $90 \mathrm{~min}$ with a cold pack $\left(\right.$ at $0^{\circ} \mathrm{C}$ ). Proteins were then stored in $5 \%$ bovine serum albumin blocking solution for $1 \mathrm{~h}$ at $25^{\circ} \mathrm{C}$ and incubated overnight at $40^{\circ} \mathrm{C}$ with monoclonal rabbit anti-ERK1/2 (dilution, 1:1,000), monoclonal rabbit anti-phospo-ERK1/2 (dilution, 1:1,000) or polyclonal rabbit anti-EAAT-2 antibodies (dilution, 1:1,000) (Cell Signaling Technology, Inc., Danvers, MA, USA). Protein was then incubated with horseradish peroxidase-labeled anti-rabbit secondary antibodies (dilution, 1:100; Hua-Mei Biotech, Beijing, China) for $1 \mathrm{~h}$ at room temperature with blocking buffer. The membrane was then visualized using the enhanced chemiluminescence kit (GE Healthcare) for $5 \mathrm{~min}$ and exposed to Kodak film (Kodak) for 5-30 seconds. GAPDH or $\beta$-actin was used as a loading control.

Statistical analysis. SPSS version 15.0 (SPSS Inc, Chicago, IL, USA) was used to perform data analysis. Neurological deficit scores and infarct volumes between the MCAO and exercise with MCAO groups were compared using Student's t-test. Relative image density among the three groups analyzed using a one-way analysis of variance was followed by a least significant difference post-hoc test. Data are presented as the mean \pm standard deviation. $\mathrm{P}<0.05$ was considered to indicate a statistically significant difference between values.

\section{Results}

Physiological variables are unchanged between the groups. Physiological parameters were evaluated in rats from each 


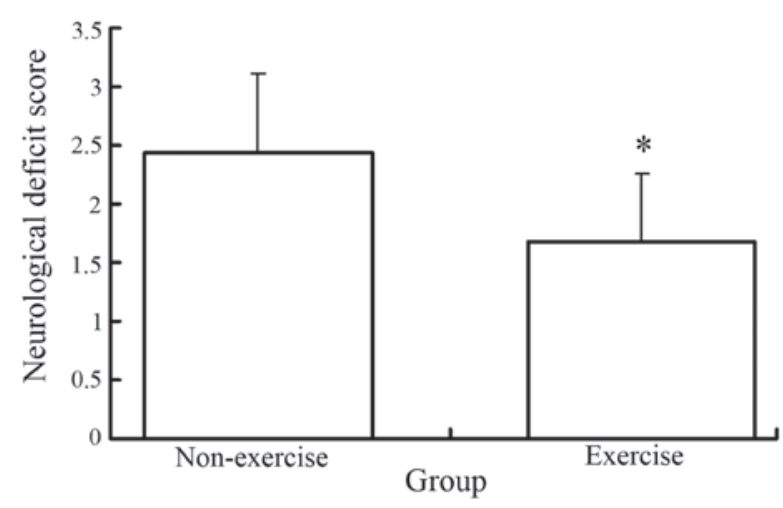

Figure 1. Neurological deficit scores of the MCAO group and exercise with MCAO group $24 \mathrm{~h}$ post-reperfusion. Data are presented as the mean \pm standard deviation ( $n=6)$. ${ }^{*} \mathrm{P}<0.05$ vs. MCAO group without exercise. MCAO, middle cerebral artery occlusion.

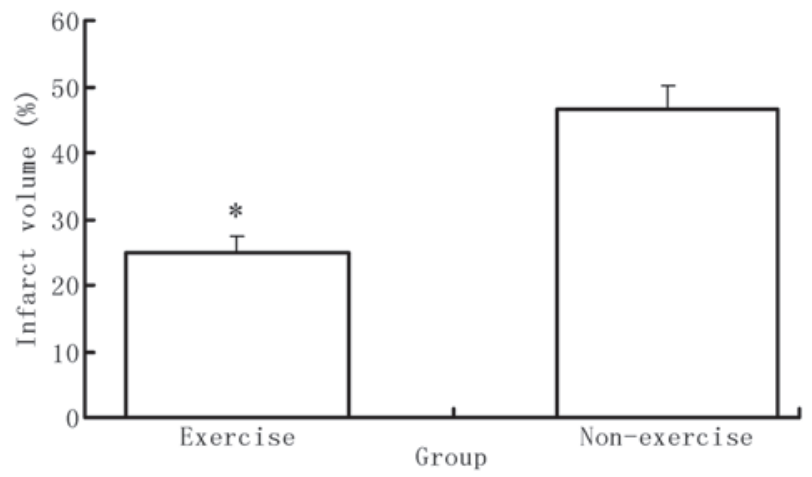

Figure 2. Difference in infarct volume between exercise with MCAO and the MCAO groups $24 \mathrm{~h}$ post-reperfusion. Data are presented as the mean \pm standard deviation. "P<0.05 vs. MCAO group without exercise. MCAO, middle cerebral artery occlusion.

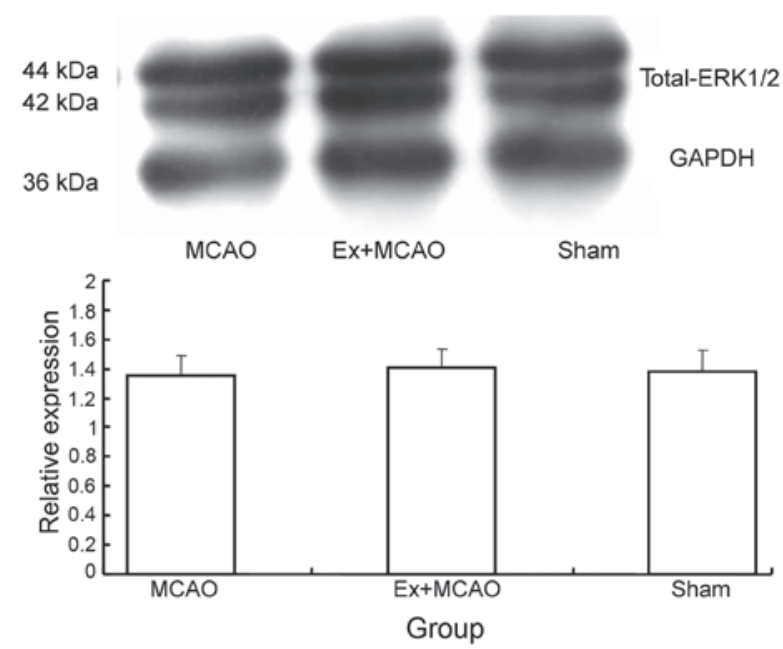

Figure 3. Relative expression of total ERK1/2. Western blot analysis of total-ERK1/2 among the three groups. GAPDH was used as the internal control. Data are presented as the mean \pm standard deviation. MCAO, middle cerebral artery occlusion; ERK, extracellular signal-regulated kinase; Ex, exercise.

group (data not shown). The parameters investigated included hydrogen ion concentration, partial pressure of carbon dioxide in the arteries and partial pressure of oxygen in the arterial
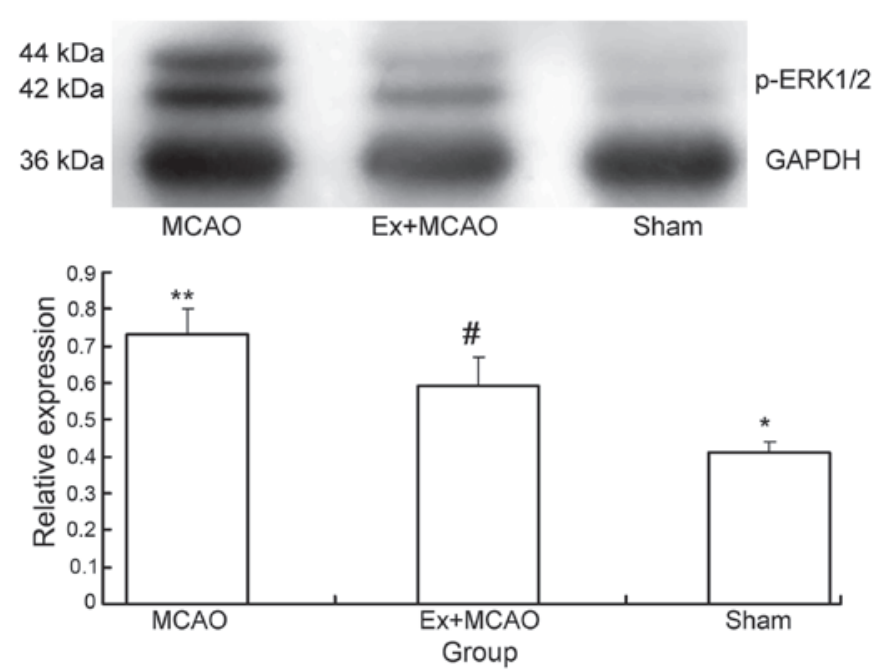

Figure 4. Relative expression of p-ERK1/2. Western blot analysis of the expression of p-ERK1/2 among the three groups. GAPDH was used as the internal control. Data are presented as the mean \pm standard deviation. ${ }^{~} \mathrm{P}<0.05$ vs. sham surgery; ${ }^{* *} \mathrm{P}<0.05$ vs. MCAO with exercise; $\mathrm{P}<0.05$ vs. MCAO without exercise groups. MCAO, middle cerebral artery occlusion; p-ERK, phosphorylated extracellular signal-regulated kinase; Ex, exercise.

$64 \mathrm{kDa}$
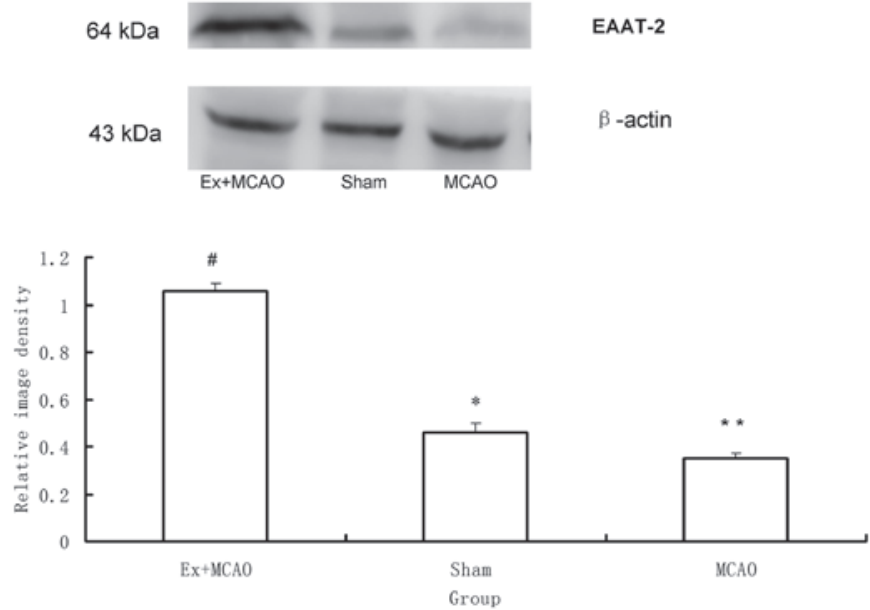

Figure 5. Difference in EAAT-2 protein expression. Western blot analysis of EAAT-2 protein expression among the three groups. $\beta$-actin was used as the internal control. Data are presented as the mean \pm standard deviation. ${ }^{~} \mathrm{P}<0.05$ vs. sham surgery; ${ }^{* *} \mathrm{P}<0.05$ vs. MCAO with exercise; ${ }^{*} \mathrm{P}<0.05$ vs. MCAO without exercise groups. MCAO, middle cerebral artery occlusion; EAAT-2, excitatory amino acid transporter; Ex, exercise.

blood. No significant differences were identified among the parameters of the the three groups $(\mathrm{P}>0.05)$.

Exercise reduces post-reperfusion neurological deficits. Neurological evaluations were performed $24 \mathrm{~h}$ post-reperfusion and their scores calculated. Rats in the sham surgery group demonstrated no neurological symptoms; however, the neurological deficit scores for the exercise with MCAO group were significantly lower than those of the MCAO group $(\mathrm{P}<0.05)$ (Fig. 1).

Exercise reduces infarct volume following MCAO surgery. Following neurological evaluation, six rats from each group were sacrificed in order to calculate the infarct volume. No 
ischemic areas were identified in the brains of rats in the sham group; however, rats of the exercise with MCAO group showed significantly decreased volumes of infarction compared with those of the MCAO group $(\mathrm{P}<0.05)$ (Fig. 2).

Exercise reduces phospho-ERK1/2 overexpression following ischemic brain injury. Western blot analysis was used to determine the protein levels of ERK1/2 and phospho-ERK1/2 in rats from each group. As shown in Figs. 3 and 4, total ERK1/2 expression was not significantly different among the three groups; however, there were significant differences in phospho-ERK1/2 expression levels among the three groups. MCAO rats demonstrated increased phospho-ERK1/2 expression compared to that of the sham surgery group $(\mathrm{P}<0.05)$; in addition, the pre-ischemic exercise group showed decreased phospho-ERK1/2 compared to that of the MCAO group $(\mathrm{P}<0.05)$, indicating that pre-ischemic exercise alleviated this effect.

EAAT-2. As shown in Fig. 5, western blot analysis revealed significant differences in EAAT-2 protein expression among the three groups $(\mathrm{P}<0.05)$. EAAT-2 was markedly decreased in the MCAO group compared to that of the sham surgery group and was markedly increased in the pre-ischemic exercise group compared to that of the MCAO group $(\mathrm{P}<0.05)$.

\section{Discussion}

Ischemic stroke is a prevalent cause of mortality in Western countries (17); therefore, studies into the alleviation of brain damage following ischemic stroke are essential. A previous study investigated a series of intervention methods and reported that physical exercise may be effective in alleviating brain injury following ischemic stroke (18); however, the mechanisms underlying the neuroprotective effects of exercise prior to ischemic brain injury required elucidation in order to encourage individuals with high risk factors of stroke to begin to exercise regularly.

In the pathological process of cerebral ischemia-reperfusion injury, neurotoxicity due to glutamate over-release is a primary damaging factor (6). EAATs were reported to have a key role in the uptake process of excess extracellular glutamate $(9,10)$, and they were found to downregulate glutamate uptake ability, resulting in an $\sim 65 \%$ increase of glutamate concentration following diffuse brain injury (19). EAAT-2 is expressed in astrocytes of different brain areas, particularly in the cerebral cortex and hippocampus (20) and was reported to be responsible for $90 \%$ of glutamate re-uptake into the prosencephalon of rats (21). EAAT-2 knockout mice showed selective neuronal deterioration in the area of hippocampal CA1, indicating the role of EAAT-2 in neuroprotection (22). The results of the present study demonstrated that pre-ischemic exercise enhanced expression levels of EAAT-2, therefore alleviating the neurotoxicity caused by excessive release of glutamate.

ERK1/2 was reported to have different roles in the pre- and post-stroke phases (11-14). Previous studies have indicated that an inhibitor of mitogen-activated protein kinase (MAPK)/ERK (MEK1) alleviated brain damage of mice following ischemic stroke, indicating that the MEK1-ERK1/2 pathway was involved in cerebral damage in the process of cerebral ischemia (13-14). Therefore, intervention which may decrease the overexpression of phospho-ERK1/2 following ischemic stroke may alleviate ischemia-induced damage.

In a previous study, the promotion of phospho-ERK1/2 induced by exercise preconditioning was found to be neuroprotective (11). Another study demonstrated that pre-ischemic exercise decreased overexpression of phospho-ERK1/2 at 48 hours following ischemia/reperfusion (8). The results of the present study further confirm that exercise preconditioning alleviated the overexpression of phospho-ERK1/2 at $24 \mathrm{~h}$ following ischemia/reperfusion.

In conclusion, the results of the present study indicated that pre-ischemic exercise exerted a neuroprotective effect via the decreased overexpression of phospho-ERK1/2 and increased expression of EAAT-2 following cerebral ischemia. Further patient studies are required in order to confirm the beneficial effect of pre-ischemic exercise in humans; however, it is encouraged that patients with a high risk of stroke exercise regularly.

\section{Acknowledgements}

The present study was supported by a grant from the National Natural Science Foundation of China (no. 81201512).

\section{References}

1. Stummer W, Baethmann A, Murr R, et al: Cerebral protection against ischemia by locomotor-activity in gerbils. Underlying mechanisms. Stroke 26: 1423-1429, 1995.

2. Ang ET, Wong PTH, Moochhala S and Ng YK: Neuroprotection associated with running: is it a result of increased endogenous neurotrophic factors? Neuroscience 118: 335-345, 2003.

3. Endres M, Gertz K, Lindauer U, et al: Mechanisms of stroke protection by physical activity. Ann Neurol 54: 582-590, 2003.

4. Li J, Luan XD, Clark JC, et al: Neuroprotection against transient cerebral ischemia by exercise pre-conditioning in rats. Neurol Res 26: 404-408, 2004.

5. Ding YH, Ding Y, Li J, et al: Exercise pre-conditioning strengthens brain microvascular integrity in a rat stroke model. Neurol Res 28: 184-189, 2006.

6. Guyot LL, Diaz FG, O'Regan MH, McLeod S, Park H and Phillis JW: Real-time measurement of glutamate release from the ischemic penumbra of the rat cerebral cortex using a focal middle cerebral artery occlusion model. Neurosci Lett 299: 37-40, 2001.

7. Zhang F, Jia J, Wu Y, Hu Y and Wang Y: The effect of treadmill training pre-exercise on glutamate receptor expression in rats after cerebral ischemia. Int J Mol Sci 11: 2658-2669, 2010.

8. Zhang F, Wu Y, Jia J and Hu YS. Pre-ischemic treadmill training induces tolerance to brain ischemia: involvement of glutamate and ERK1/2. Molecules 15: 5246-5257, 2010.

9. Beart PM and O'Shea RD. Transporters for L-glutamate: an update on their molecular pharmacology and pathological involvement. Br J Pharmacol 150: 5-17, 2007.

10. Suchak SK, Baloyianni NV, Perkinton MS, Williams RJ, Meldrum BS and Rattray M: The 'glial' glutamate transporter, EAAT2 (Glt-1) accounts for high affinity glutamate uptake into adult rodent nerve endings. J Neurochem 84: 522-532, 2003.

11. Liebelt B, Papapetrou P, Ali A, et al: Exercise preconditioning reduces neuronal apoptosis in stroke by up-regulating heat shock protein-70 (heat shock protein-72) and extracellular signal-regulated-kinase $1 / 2$. Neuroscience 166 , 1091-1100, 2010 .

12. Lu ZM and Xu SC: ERK1/2 MAP kinases in cell survival and apoptosis. IUBMB Life 58: 621-631, 2006.

13. Alessandrini A, Namura S, Moskowitz MA and Bonventre JV: MEK1 protein kinase inhibition protects against damage resulting from focal cerebral ischemia. Proc Natl Acad Sci USA 96: 12866-12869, 1999. 
14. Namura S, Iihara K, Takami S, et al: Intravenous administration of MEK inhibitor U0126 affords brain protection against forebrain ischemia and focal cerebral ischemia. Proc Natl Acad Sci USA 98: 11569-11574, 2001.

15. Longa EZ, Weinstein PR, Carlson S and Cummins R: Reversible middle cerebral artery occlusion without craniectomy in rats. Stroke 20: 84-91, 1989.

16. Ding YH, Ding Y, Li J, Bessert DA and Rafols JA: Exercise pre-conditioning strengthens brain microvascular integrity in a rat stroke model. Neurol Res 28: 184-189, 2006.

17. National Center for Health Statistics: Health, United States, 2010 With Special Feature on Death and Dying. Hyattsville (MD, USA) 2011.

18. Krarup LH, Truelsen T, Gluud C, et al; ExStroke Pilot Trial Group: Prestroke physical activity is associated with severity and long-term outcome from first-ever stroke. Neurology 71: 1313-1318, 2008.
19. Hinzman JM, Thomas TC, Quintero JE and Gerhardt GA, Lifshit J: Disruptions in the regulation of extracellular glutamate by neurons and glia in the rat striatum two days after diffuse brain injury. J Neurotrauma 29: 1197-1208, 2012.

20. Kanai Y and Hediger MA: The glutamate/neutral amino acid transporter family SLC1: molecular, physiological and pharmacological aspects. Pflugers Arch 447: 469-479, 2004.

21. Verma R, Mishra V, Sasmal D and Raghubir R: Pharmacological evaluation of glutamate transporter 1 (GLT-1) mediated neuroprotection following cerebral ischemia/reperfusion injury. Eur J Pharmacol 638: 65-71, 2010.

22. Tanaka K, Watase K, Manabe T, et al: Epilepsy and exacerbation of brain injury in mice lacking the glutamate transporter GLT-1. Science 276: 1699-1702, 1997. 